\begin{tabular}{cc} 
Sharif University of Technology \\
Scientia Iranica \\
SCIENTIA & Transactions F: Nanotechnology \\
I RAN I CA & \\
\hline
\end{tabular}

\title{
On the oscillatory frequency of the carbon nanotube-based nanothermometers
}

\author{
R. Ansari*, M. Daliri and M. Hosseinzadeh* \\ Department of Mechanical Engineering, University of Guilan, Rasht, P.O. Box 3756, Iran. \\ Received 18 June 2015; received in revised form 5 April 2016; accepted 26 November 2016
}

\section{KEYWORDS \\ Electromechanical nanothermometers; \\ Carbon nanotubes; \\ Continuum \\ approximation; \\ Oscillation frequency.}

\begin{abstract}
Electromechanical nanothermometers are instruments that work on the basis of the van der Waals (vdW) potential energy and interaction force of their constituent carbon nanotubes (CNTs). The CNT-based nanothermometers have two different configurations: telescope and shuttle configurations. In this article, based on the Lennard-Jones potential function together with the continuum approximation, first, the vdW potential energy and interaction force for a telescope configuration with finite CNTs are derived, which have not been obtained in the previous research studies. Thereafter, by employing the interaction force, the equation of motion between constituent CNTs is solved. Subsequently, a new semi-analytical expression is obtained which enables one to precisely evaluate the oscillation frequency. By employing the given formulae, effects of different system parameters on the vdW interactions and oscillation frequency are shown.

(C) 2017 Sharif University of Technology. All rights reserved.
\end{abstract}

\section{Introduction}

Nanotechnology is one the most promising technologies which has been evolving with a rapid pace in recent years. The advances in nanotechnology have been growing even faster when the landmark paper of Iijima on carbon nanotubes (CNTs) was published in 1991 [1]. Due to incomparable mechanical and electronic properties, CNTs are recognized as the main building blocks for various nanotechnology applications [2,3]. Gigahertz (GHz) oscillators [4-6] are one of the most versatile types of such devices used in ultra-fast optical filters and ultra-sensitive nano-antennas $[7,8]$. The idea of such devices was proposed by Cumings and Zettl [9] via conducting experiments on two nested Multi-Walled Carbon Nanotubes (MWCNTs) which had nearly frictionless telescopic motion. In their experiment, one end of the outer shell was opened and the core was pulled out by a movable manipulator

*. Corresponding authors.

E-mail addresses: r_ansari@guilan.ac.ir (R. Ansari); mhdshhosseinzadeh@gmail.com (M. Hosseinzadeh) in a high-resolution Transmission Electron Microscopy (TEM).

The walls of adjacent tubes in MWCNTs are held together via weak van der Waals (vdW) forces that are inter-atomic forces between non-bonded atomic pairs. These forces generate sliding and rotary motions between the walls of MWCNTs. The oscillatory behavior and vdW interactions of CNTs have been a subject of wide interest, and numerous studies have been devoted to investigate their different features thus far [10-17].

Understanding the properties of materials at the nanoscale requires sensitive devices which can provide measurements of high precision, e.g. nanothermometer [18-23]. Nanothermometer is used to measure temperatures of spaces surrounding the nanoscale devices [24-29]. These instruments overcome the difficulty encountered in measuring temperature of nanoscale systems via thermometer and thermocouple. Nanothermometer can operate in conditions with high temperature changes where conventional measurement systems, such as thermometer and thermocouple, almost fail. Carbon nanothermometer was first proposed by Gao et al. [30] who accidently discovered that 
CNTs filled with gallium can be used as a device to measure temperature between $50^{\circ} \mathrm{C}$ to $500^{\circ} \mathrm{C}$. Liu et al. [31] examined the freezing and melting characteristics of gallium encapsulated in CNTs through insitu observation in a TEM. Their study showed that the state of gallium encapsulated in CNTs is liquid up to $-80^{\circ} \mathrm{C}$. The precision of measuring through gallium-filled CNT nanothermometer is dependent on its identification and calibration in a TEM which can impose limitations on its applicability. There are also some other recently-published research papers on nanotermometry. For instance, Liu et al. [32] investigated the fluorescent nanothermometer which has the potential to monitor the temperature variation in the nanoregime via biocompatible fluorescent carbon nanodots through one-step microwave assisted synthesis and two types of polymers. Pi and coworkers [33] studied the magnetic nanothermometry utilizing both odd and even harmonics of magnetic nanoparticles under low frequency $(f=117 \mathrm{~Hz})$ ac and dc magnetic fields on the basis of the first-order Langevin function. Mayle and associates [34] performed investigations on the thermometry of CNT circuits by monitoring the intrinsic temperature and the thermal management for the CNT and nano-circuits. Su et al. [35] fabricated Ga-filled $\mathrm{MgO}$ and silica nanotubes by a simple onestep method. Zhou et al. [36] reviewed the principles and aspects of nanothermometer design driven by two emerging areas: single-cell thermogenesis and imageguided thermal treatments. Also, Bichoutskaia et al. [37] introduced another type of CNT nanothermometer which consists of DWCNTs with nonchiral commensurate walls. These authors investigated the dependence of the inter-wall interaction energy on the relative displacements of the walls of a DWCNT constituting a $(6,6)$ and a $(11,11)$ CNT. This type of nanothermometer has two common forms: shuttle and telescope configurations. Shuttle nanothermometer has a movable outer wall and a fixed inner wall, whereas the other one has a movable inner wall and two fixed outer walls. Different aspects of these nanothermometers were investigated in [37-39]. Studies performed so far on this topic clearly show that the vdW interaction and relative motions of DWCNTs have significant influence on the performance of such devices $[38,39]$. In this respect, Rahmat et al. [38] and Ansari et al. [39] examined the vdW potential energy and interaction force between constituent CNTs of nanothermometer with shuttle and telescope configurations. Both of these authors employed continuum approximation along with the Lennard-Jones (LJ) function to model the vdW interactions.

The aim of the present paper is to investigate the oscillatory behavior of the CNT nanothermometer. In particular, in order to estimate the interaction between two nanostructures via continuum approximation, sur- face integrals must be carried out over both smeared surfaces. The resulting integral is a quadruple integral which can be reduced to a double one by means of linear transformation. This double integral finally turns into a single integral via mathematical manipulation that can be readily used to calculate potential energy and interaction force. Further, the oscillatory behavior of movable CNTs of nanothermometers is analyzed through a new semi-analytical expression obtained from the force equation.

\section{Interaction of constituent CNTs of nanothermometers}

In this section, the derivation of the $\mathrm{vdW}$ interaction force is briefly described which is later used to extract the associated expression for oscillatory frequency. The total potential energy is achieved by summing the potential energy between the pair of atoms from each molecule as follows:

$$
\phi^{\text {tot }}=\sum_{i} \sum_{j} \Phi\left(\rho_{i j}\right) \text {. }
$$

In the preceding equation, $\Phi\left(\rho_{i j}\right)$ is the LJ potential function for atoms $i$ and $j$ at distance $\rho_{i j}$ apart. The LJ potential function is expressed as follows:

$$
\Phi\left(\rho_{i j}\right)=-\frac{A}{\rho_{i j}^{6}}+\frac{B}{\rho_{i j}^{12}},
$$

in which $A$ and $B$ indicate the attractive and repulsive constants, respectively. According to the continuum approximation, carbon atoms are assumed to be uniformly distributed over the surfaces of interacting entities. So, the double summation in Eq. (1) can be replaced by a double integral as follows [11]:

$$
\phi^{t o t}=n_{1} n_{2} \iint \Phi(\rho) d \sum_{1} d \sum_{2},
$$

where $n_{1}$ and $n_{2}$ represent the mean surface densities of atoms on the first and second molecules, respectively, and $\rho$ indicates the distance between two typical surface elements $d \sum_{1}$ and $d \sum_{2}$ on each molecule. In addition, by taking the negative gradient of the potential energy, the $\mathrm{vdW}$ interaction force is attained as follows:

$$
F_{v d W}=-\nabla \phi^{t o t}
$$

\subsection{The VdW interactions for telescope configurations with finite CNTs}

In this part, the derivation of the vdW potential energy and interaction force equations for a telescope nanothermometer with finite constituent CNTs is discussed. It is assumed that the lengths of the inner CNT, righthand side CNT, and left-hand side CNT are equal to 
$2 L_{1}, h$, and $H$, respectively. According to Figure 1, the origin of the reference coordinate system is located at the middle of the two outer CNTs. In addition, the distance of the middle of the inner CNT from the origin is shown by $Z$. The parametric equations for a typical point on the surface of the left-hand side outer CNT, the inner CNT, and the right-hand side outer CNT can be represented by $\left(R_{3} \cos \theta_{2}, R_{3} \sin \theta_{2}, z_{3}\right)$, $\left(R_{1} \cos \theta_{1}, R_{1} \sin \theta_{1}, z_{1}\right)$, and $\left(R_{2} \cos \theta_{2}, R_{2} \sin \theta_{2}, z_{2}\right)$, respectively, where $R$ is the radius of CNT. Thus, the $\mathrm{vdW}$ potential energy for the inner and right-hand tubes can be obtained as follows:

$$
\begin{aligned}
\phi_{12}= & R_{1} R_{2} n_{c}^{2} \int_{0}^{2 \pi} \int_{0}^{2 \pi} \int_{Z-L_{1}}^{Z+L_{1}} \int_{L_{2}}^{L_{2}+h} \\
& \left(\frac{-A}{\rho^{6}}+\frac{B}{\rho^{12}}\right) d z_{2} d z_{1} d \theta_{2} d \theta_{1}
\end{aligned}
$$

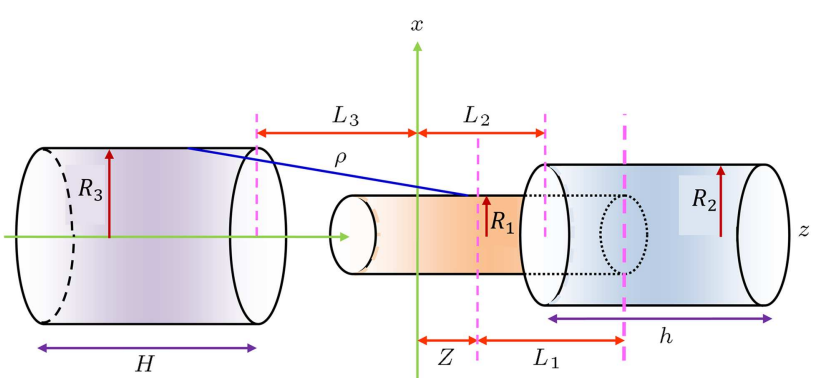

Figure 1. Schematic of telescope configuration consisting of finite tubes. where $n_{c}$ represents the mean surface density of carbon atom, and:

$$
\rho_{12}^{2}=R_{1}^{2}+R_{2}^{2}-2 R_{1} R_{2} \cos \left(\theta_{1}-\theta_{2}\right)+\left(z_{1}-z_{2}\right)^{2} .
$$

The aforementioned quadrature integral can be reduced to a double integral through linear transformations performed on $\theta_{1}-\theta_{2}$ and $z_{1}-z_{2}$ planes, as illustrated in Figure 2:

$$
\begin{aligned}
& \begin{cases}z_{1}-z_{2}=u \Rightarrow z_{1}=\frac{u+v}{2} & \Rightarrow j(u, v)=\frac{1}{2} \\
z_{1}+z_{2}=v \Rightarrow z_{2}=\frac{v-u}{2} & \Rightarrow d z_{1} d z_{2}=\frac{1}{2} d u d v\end{cases} \\
& \begin{cases}\theta_{1}-\theta_{2}=\alpha \Rightarrow \theta_{1}=\frac{\alpha+\beta}{2} & \Rightarrow j(\alpha, \beta)=\frac{1}{2} \\
\theta_{1}+\theta_{2}=\beta \Rightarrow \theta_{2}=\frac{\beta-\alpha}{2} & \Rightarrow d \theta_{1} d \theta_{2}=\frac{1}{2} d \alpha d \beta\end{cases}
\end{aligned}
$$

Accordingly, one can write:

$$
\phi_{12}=\gamma_{12} \iint_{A} \iint_{S} \varphi\left(z_{1}-z_{2}, \theta_{1}-\theta_{2}\right) d z_{2} d z_{1} d \theta_{2} d \theta_{1},
$$

and:

$$
\phi_{12}=\gamma_{12} \int_{A^{\prime}} \iint_{S^{\prime}} \frac{1}{4} \varphi(u, \alpha) d v d u d \beta d \alpha .
$$
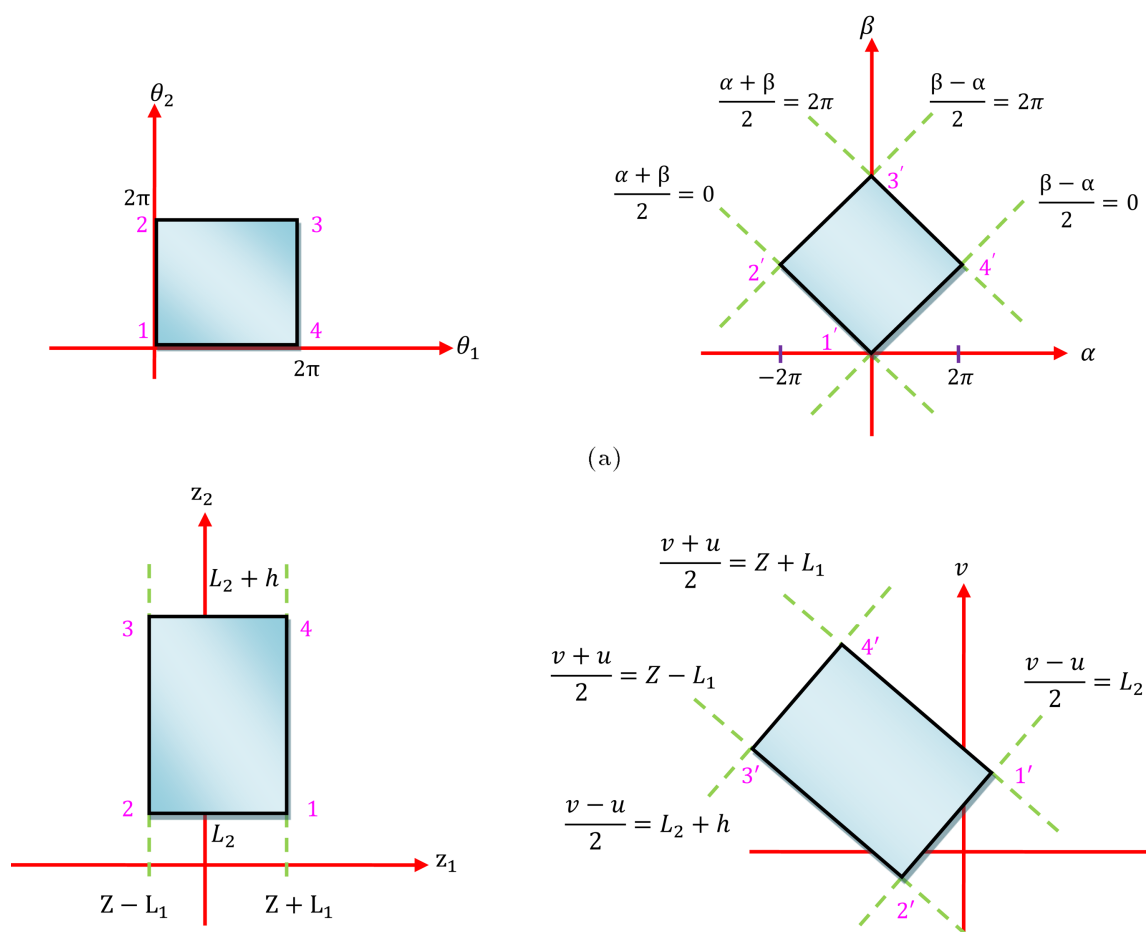

(a)

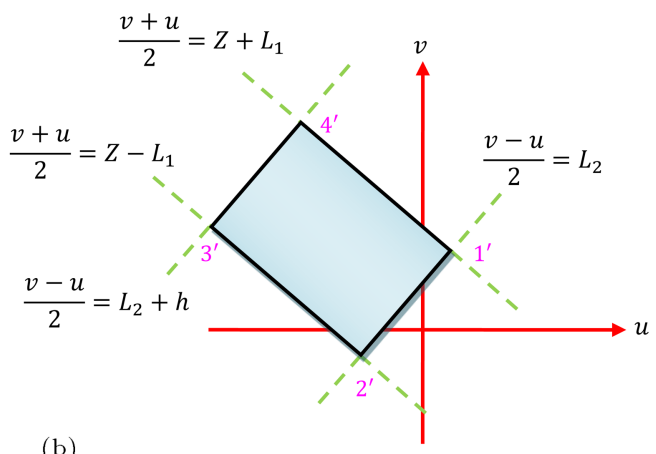

(b)

Figure 2. (a) Linear transformation from plane $\theta_{1}-\theta_{2}$ to plane $\alpha-\beta$. (b) Linear transformation from plane $z_{1}-z_{2}$ to plane $u-v$. 
So:

$$
\phi_{12}^{t o t}=\gamma_{12} \int_{A^{\prime}} \int_{S^{\prime}} \int_{4} \frac{1}{4} \varphi(u, \alpha) d v d u d \beta d \alpha
$$

where $\gamma_{12}=R_{1} R_{2} n_{c}^{2}$. Now, by employing the abovementioned transformations, Eq. (9) becomes:

$$
\begin{aligned}
\phi_{12}^{t o t}= & \gamma_{12} \int_{-2 \pi}^{0} \int_{-\alpha}^{4 \pi+\alpha} \frac{1}{2} \Gamma(\alpha)_{12} d \beta d \alpha \\
& +\gamma_{12} \int_{0}^{2 \pi} \int_{\alpha}^{4 \pi-\alpha} \frac{1}{2} \Gamma(\alpha)_{12} d \beta d \alpha .
\end{aligned}
$$

Thus:

$$
\begin{aligned}
\phi_{12}^{\text {tot }}= & \gamma_{12} \int_{-2 \pi}^{0}(2 \pi+\alpha) \Gamma(\alpha)_{12} d \alpha \\
& +\gamma_{12} \int_{0}^{2 \pi}(2 \pi-\alpha) \Gamma(\alpha)_{12} d \alpha .
\end{aligned}
$$

It is remarkable to note that $\Gamma(\alpha)_{12}$ is an even function in terms of $\alpha$, so Eq. (11) turns into:

$$
\phi_{12}^{t o t}=2 \gamma_{12} \int_{0}^{2 \pi}(2 \pi-\alpha) \Gamma(\alpha)_{12} d \alpha
$$

where:

$$
\begin{aligned}
\Gamma(\alpha)_{12}= & \int_{u_{3}}^{u_{4}} \int_{2\left(Z-L_{1}\right)-u}^{2\left(L_{2}+h\right)+u} \frac{1}{2} \phi(\alpha, u) d v d u \\
& +\int_{u_{4}}^{u 2} \int_{2\left(Z-L_{1}\right)-u}^{2\left(Z+L_{1}\right)-u} \frac{1}{2} \phi(\alpha, u) d v d u \\
& +\int_{u_{2}}^{u_{1}} \int_{2 L_{2}+u}^{2\left(Z+L_{1}\right)-u} \frac{1}{2} \phi(\alpha, u) d v d u
\end{aligned}
$$

It is worth mentioning that $\phi$ is independent of $v$, so:

$$
\begin{aligned}
\Gamma(\alpha)_{12}= & \int_{u_{3}}^{u_{4}}\left(u-u_{3}\right) \phi(\alpha, u) d u \\
& +\int_{u_{4}}^{u_{2}}\left(u_{1}-u_{2}\right) \phi(\alpha, u) d u \\
& +\int_{u_{2}}^{u_{1}}\left(u_{1}-u\right) \phi(\alpha, u) d u,
\end{aligned}
$$

in which:

$$
\begin{aligned}
& u_{1}=Z+\left(L_{1}-L_{2}\right), \quad u_{2}=Z-\left(L_{1}+L_{2}\right), \\
& u_{3}=Z-\left(L_{1}+L_{2}+h\right) \quad \text { and } \\
& u_{4}=Z+\left(L_{1}-L_{2}-h\right)
\end{aligned}
$$

Therefore, the following relation can be obtained for $\Gamma(\alpha)_{12}$ :

$$
\begin{aligned}
\Gamma(\alpha)_{12}= & \sum_{k=1}^{2} H_{k}\left\{\sum_{m=1}^{4}\left(\frac{(-1)^{m+1}}{2(3 k-1)}\left[\frac{1}{\left(u_{m}^{2}+\lambda^{2}\right)^{(3 k-1)}}\right]\right)\right. \\
& +\sum_{m=1}^{4} G_{1}^{(k)} \frac{(-1)^{m+1}}{\lambda^{6 k-1}} u_{m} \tan ^{-1}\left(\frac{u_{m}}{\lambda}\right) \\
& \left.+\sum_{m=1}^{4} \sum_{n=1}^{3 k-1}(-1)^{m+1} G_{n}^{(k)} \frac{u_{(m)}^{2} \lambda^{2(n-3 k)}}{\left(\lambda^{2}+u_{m}^{2}\right)^{n}}\right\} .
\end{aligned}
$$

The vdW potential energy and interaction force between the inner and outer left-hand side tubes can be achieved as follows:

$$
\begin{aligned}
\phi_{13}= & R_{1} R_{3} n_{c}^{2} \int_{0}^{2 \pi} \int_{0}^{2 \pi} \int_{Z-L_{1}}^{Z+L_{1}} \int_{-\left(L_{3}+H\right)}^{-L_{3}} \\
& \left(\frac{-A}{\rho^{6}}+\frac{B}{\rho^{12}}\right) d z_{3} d z_{1} d \theta_{3} d \theta_{1},
\end{aligned}
$$

where $\rho_{13}^{2}=R_{1}^{2}+R_{3}^{2}-2 R_{1} R_{3} \cos \left(\theta_{1}-\theta_{3}\right)+\left(z_{1}-z_{3}\right)^{2}$.

Following the same procedure as described for the interactions between the inner and the right-hand side tubes, one obtains:

$$
\begin{aligned}
\Gamma(\alpha)_{13}= & \int_{u_{2}}^{u_{1}}\left(u-u_{2}\right) \phi(\alpha, u) d u \\
& +\int_{u_{1}}^{u_{3}}\left(u_{4}-u_{3}\right) \phi(\alpha, u) d u \\
& +\int_{u_{3}}^{u_{4}}\left(u_{4}-u\right) \phi(\alpha, u) d u
\end{aligned}
$$

where:

$$
\begin{aligned}
& u_{1}=Z+\left(L_{1}+L_{3}\right), \\
& u_{2}=Z-\left(L_{1}-L_{3}\right), \\
& u_{3}=Z-\left(L_{1}-L_{3}-H\right), \\
& u_{4}=Z+\left(L_{1}+L_{3}+H\right),
\end{aligned}
$$

and as a result, the following expression can be obtained:

$$
\Gamma(\alpha)_{13}=\sum_{k=1}^{2} H_{k}\left\{\sum_{m=1}^{4}\left(\frac{(-1)^{m}}{2(3 k-1)}\left[\frac{1}{\left(u_{m}^{2}+\lambda^{2}\right)^{(3 k-1)}}\right]\right)\right.
$$

$$
\begin{aligned}
& +\sum_{m=1}^{4} G_{1}^{(k)} \frac{(-1)^{m}}{\lambda^{6 k-1}} u_{m} \tan ^{-1}\left(\frac{u_{m}}{\lambda}\right) \\
& \left.+\sum_{m=1}^{4} \sum_{n=1}^{3 k-1}(-1)^{m} G_{n}^{(k)} \frac{u_{(m)}^{2} \lambda^{2(n-3 k)}}{\left(\lambda^{2}+u_{m}^{2}\right)^{n}}\right\},
\end{aligned}
$$


where:

$$
\lambda^{2}=R_{1}^{2}+R_{3}^{2}-2 R_{1} R_{3} \cos \alpha,
$$

and:

$$
\begin{aligned}
& H_{1}=-A, \quad H_{2}=B, \\
& G_{1}^{(1)}=\frac{3}{8}, \quad G_{2}^{(1)}=\frac{1}{4}, \\
& G_{1}^{(2)}=\frac{63}{256}, \quad G_{2}^{(2)}=\frac{21}{128}, \quad G_{3}^{(2)}=\frac{21}{160}, \\
& G_{4}^{(2)}=\frac{9}{80}, \quad G_{5}^{(2)}=\frac{1}{10}, \\
& G_{b}^{(1)}=\frac{3 \pi}{4}, \quad G_{b}^{(2)}=\frac{63 \pi}{128} .
\end{aligned}
$$

Thus, the total potential energy for the telescope configuration can be obtained by:

$$
\phi^{t o t}=\phi_{12}+\phi_{13}
$$

Additionally, the total vdW interaction force can be obtained as follows:

$$
F_{Z}^{\text {tot }}=\left(F_{Z}\right)_{12}+\left(F_{Z}\right)_{13} .
$$

The vdW interaction force $\left(F_{Z}\right)_{12}$ can be obtained by:

$$
\left(F_{Z}\right)_{12}=-\frac{\partial \phi_{12}^{\text {tot }}}{\partial Z}=-2 \gamma_{12} \int_{0}^{2 \pi}(2 \pi-\alpha) \frac{\partial \Gamma(\alpha)_{12}}{\partial Z} d \alpha
$$

where:

$$
\begin{aligned}
\frac{\partial \Gamma(\alpha)_{12}}{\partial Z}= & \sum_{k=1}^{2} H_{k}\left\{\sum_{m=1}^{4}\left((-1)^{(m+2)}\left[\frac{u_{m}}{\left(u_{m}^{2}+\lambda^{2}\right)^{3 k}}\right]\right)\right. \\
& +\sum_{m=1}^{4} G_{1}^{(k)} \frac{(-1)^{(m+1)}}{\lambda^{6 k-1}}\left[\tan ^{-1}\left(\frac{u_{m}}{\lambda}\right)\right. \\
& \left.+\frac{\lambda}{\left(u_{m}^{2}+\lambda^{2}\right)} u_{m}\right]+2 \sum_{m=1}^{4} \sum_{n=1}^{3 k-1}(-1)^{(m+1)} \\
& G_{n}^{(k)} \lambda^{2(n-3 k)}\left[\frac{u_{m}}{\left(u_{m}^{2}+\lambda^{2}\right)^{n}}\right. \\
& \left.\left.-\frac{n}{\left(\lambda^{2}+u_{m}^{2}\right)^{n+1}} u_{(m)}^{3}\right]\right\}
\end{aligned}
$$

and:

$$
\lambda^{2}=R_{1}^{2}+R_{2}^{2}-2 R_{1} R_{2} \cos \alpha .
$$

The vdW interaction force between the inner and left-hand side tubes becomes:

$$
F_{13}^{t o t}=-\frac{\partial \phi_{13}^{t o t}}{\partial Z}=-2 \gamma_{13} \int_{0}^{2 \pi}(2 \pi-\alpha) \frac{\partial \Gamma(\alpha)_{13}}{\partial Z} d \alpha,
$$

where:

$$
\begin{aligned}
\frac{\partial \Gamma(\alpha)_{13}}{\partial Z} & =-\frac{\partial \Gamma(\alpha)_{12}}{\partial Z} \\
& =\sum_{k=1}^{2} H_{k}\left\{\sum_{m=1}^{4}\left((-1)^{m+1}\right)\left[\frac{u_{m}}{\left(u_{m}^{2}+\lambda^{2}\right)^{3 k}}\right]\right) \\
& +\sum_{m=1}^{4} G_{1}^{(k)} \frac{(-1)^{m}}{\lambda^{6 k-1}}\left[\tan ^{-1}\left(\frac{u_{m}}{\lambda}\right)\right. \\
& \left.+\frac{\lambda}{\left(u_{m}^{2}+\lambda^{2}\right)} u_{m}\right]+2 \sum_{m=1}^{4} \sum_{n=1}^{3 k-1}(-1)^{m} \\
& G_{n}^{(k)} \lambda^{2(n-3 k)}\left[\frac{u_{m}}{\left(u_{m}^{2}+\lambda^{2}\right)^{n}}\right. \\
& \left.\left.-\frac{n}{\left(\lambda^{2}+u_{m}^{2}\right)^{n+1}} u_{(m)}^{3}\right]\right\} .
\end{aligned}
$$

\subsection{VdW interactions for shuttle}

configurations consisting of finite CNTs

The geometric illustration of a shuttle configuration with finite inner tube is shown in Figure 3. The inner and outer tubes are respectively of lengths $2 L_{1}$ and $2 L_{2}$ and radii $b_{1}$ and $b_{2}$. In addition, the origin of the reference frame is located at the midpoint of the inner tube. It was discussed in [39] that the vdW potential energy between the inner and outer tubes can be evaluated from the following single integral equation:

$$
\phi^{t o t}=2 \gamma \int_{0}^{2 \pi}(2 \pi-\alpha) \Gamma(\alpha) d \alpha
$$

in which $\gamma=b_{1} b_{2} n_{c}^{2}$, and:

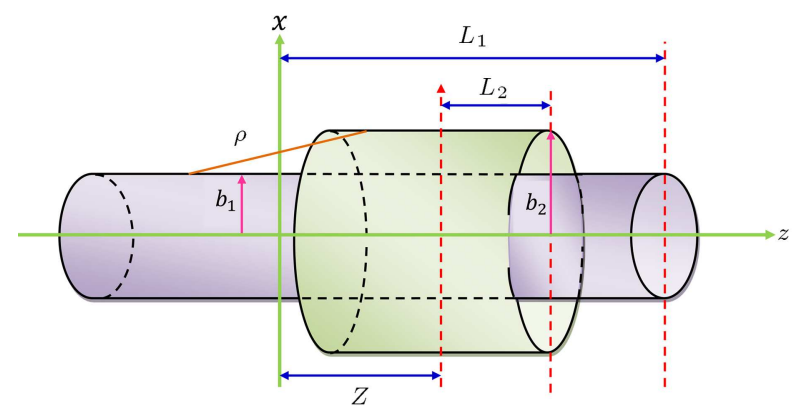

Figure 3. Schematic of shuttle configuration consisting of two finite CNTs. 


$$
\begin{aligned}
\Gamma(\alpha)= & \sum_{k=1}^{2} H_{k}\left\{\sum_{m=1}^{4}\left(\frac{(-1)^{m}}{2(3 k-1)}\left[\frac{1}{\left(u_{m}^{2}+\lambda^{2}\right)^{(3 k-1)}}\right]\right)\right. \\
& +\sum_{m=1}^{4} G_{1}^{(k)} \frac{(-1)^{m}}{\lambda^{6 k-1}} u_{m} \tan ^{-1}\left(\frac{u_{m}}{\lambda}\right) \\
& \left.+\sum_{m=1}^{4} \sum_{n=1}^{3 k-1}(-1)^{m} G_{n}^{(k)} \frac{u_{(m)}^{2} \lambda^{2(n-3 k)}}{\left(\lambda^{2}+u_{m}^{2}\right)^{n}}\right\},
\end{aligned}
$$

where:

$$
\begin{array}{ll}
\lambda^{2}=b_{1}^{2}+b_{2}^{2}-2 b_{1} b_{2} \cos \alpha, & \\
u_{1}=-Z+\left(L_{2}-L_{1}\right), & u_{2}=-Z-\left(L_{2}+L_{1}\right), \\
u_{3}=-Z-\left(L_{2}-L_{1}\right), & u_{4}=-Z+\left(L_{2}+L_{1}\right) .
\end{array}
$$

By differentiating the total potential energy with respect to $Z$ due to the symmetry of the problem, the axial interaction force is achieved as follows:

$$
F_{Z}^{t o t}=-\frac{\partial \Phi^{t o t}}{\partial Z}=-2 \gamma \int_{0}^{2 \pi}(2 \pi-\alpha) \frac{\partial \Gamma(\alpha)}{\partial Z} d \alpha,
$$

where:

$$
\begin{aligned}
\frac{\partial \Gamma(\alpha)}{\partial Z}= & \sum_{k=1}^{2} H_{k}\left\{\sum_{m=1}^{4}\left((-1)^{(m+2)}\left[\frac{u_{m}}{\left(u_{m}^{2}+\lambda^{2}\right)^{3 k}}\right]\right)\right. \\
& +\sum_{m=1}^{4} G_{1}^{(k)} \frac{\left.(-1)^{m+1}\right)}{\lambda^{6 k-1}}\left[\tan ^{-1}\left(\frac{u_{m}}{\lambda}\right)\right. \\
& \left.+\frac{\lambda}{\left(u_{m}^{2}+\lambda^{2}\right)} u_{m}\right]+2 \sum_{m=1}^{4} \sum_{n=1}^{3 k-1}(-1)^{(m+1)} \\
& G_{n}^{(k)} \lambda^{2(n-3 k)}\left[\frac{u_{m}}{\left(u_{m}^{2}+\lambda^{2}\right)^{n}}\right. \\
& \left.\left.-\frac{n}{\left(\lambda^{2}+u_{m}^{2}\right)^{n+1}} u_{m}^{3}\right]\right\} .
\end{aligned}
$$

\section{Equation of motion and frequency of the oscillation}

By making use of the Newton's second law, the equation of motion for the inner tube can be expressed as follows [11]:

$$
M \frac{d^{2} Z}{d t^{2}}=F_{Z}^{v d w}
$$

where $M$ is the mass of the movable tube and can be evaluated as follows [11]:

$$
\begin{aligned}
& M=\left(\frac{4 \pi \sqrt{3}}{9}\right) \eta(4 b L) a^{-2} m_{0}, \\
& \eta=r_{c}\left[1-\frac{\left(r_{c}-1\right) t}{2 b}\right] \quad t=0.34 \mathrm{~nm},
\end{aligned}
$$

where $a=1.42 \AA$ indicates the carbon-carbon bond length, $L$ is the half length of movable tube, $b$ denotes the radius of movable tube, $m_{0}$ is the mass of a single carbon atom, $t$ indicates the inter-shell spacing, and $r_{c}$ denotes the number of inner shells.

The frequency of oscillation can be achieved from the equation of motion or from the energy equation. As long as the frictional effect is ignorable, the total energy remains unchanged Thus, according to the conservation of the mechanical energy, the following relation can be obtained [11]:

$$
\frac{M}{2} \dot{Z}^{2}+E(Z)=E\left(A_{0}\right),
$$

where $A_{0}$ indicates the amplitude of the oscillation.

As discussed in [39], among the terms appeared in the potential energy and $\mathrm{vdW}$ interaction force formulae for both of shuttle and telescope configurations, the dominant terms are those containing $\tan ^{-1}$. It should be noted that since the telescope nanothermometer with finite tubes is a special case of telescope nanothermometer with two infinite outer tubes, one can conclude that the series consisting of $\tan ^{-1}$ are the dominant terms of the potential energy and $\mathrm{vdW}$ interaction force formulae for this case as well. Thus, by inserting dominant terms of Eqs. (21) and (27) into Eq. (33), the following expressions can be obtained:

$$
\begin{aligned}
\frac{M}{2} \dot{Z}^{2}= & 2 \gamma \int_{0}^{2 \pi}(2 \pi-\alpha) \sum_{k=1}^{2} H_{k} \sum_{m=1}^{4} \\
& G_{1}^{(k)} \frac{(-1)^{m}}{\lambda^{6 k-1}}\left[A_{0 m} \tan ^{-1}\left(\frac{A_{0 m}}{\lambda}\right)\right. \\
& \left.-u_{m} \tan ^{-1}\left(\frac{u_{m}}{\lambda}\right)\right] d \alpha
\end{aligned}
$$

where $A_{0 m}=A_{0}+l_{m}$. Upon the use of the elementary trigonometric identities, the statement in the bracket becomes:

$$
\begin{aligned}
A_{0 m} \tan ^{-1}\left(\frac{A_{0 m}}{\lambda}\right) & -u_{m} \tan ^{-1}\left(\frac{u_{m}}{\lambda}\right) \\
& =\left(A_{m}-Z\right) \\
& \tan ^{-1}\left(\frac{u_{m}}{\lambda}\right)+A_{0 m} \\
& \tan ^{-1}\left[\frac{\lambda\left(A_{0}-Z\right)}{\lambda^{2}+A_{0 m} u_{m}}\right] .
\end{aligned}
$$

By applying Taylor series to the second term on the right-hand side of Eq. (35), one can obtain the following expression: 


$$
\begin{aligned}
\frac{M}{4 \gamma}\left(\frac{d Z}{d t}\right)^{2}= & \left(A_{0}-Z\right) \int_{0}^{2 \pi}(2 \pi-\alpha) \sum_{k=1}^{2} H_{k} \\
& G_{1}^{(k)} \sum_{m=1}^{4} \frac{(-1)^{m}}{\lambda^{(6 k-1)}}\left\{\tan ^{-1}\left(\frac{u_{m}}{\lambda}\right)\right. \\
& +\frac{\lambda A_{0 m}}{\lambda^{2}+A_{0 m} u_{m}}\left[1-\frac{1}{3}\left(\frac{\lambda\left(A_{0}-Z\right)}{\lambda^{2}+A_{0 m} u_{m}}\right)^{2}\right. \\
& +\ldots]\} d \alpha .
\end{aligned}
$$

Now, by performing an integration over both sides of the previous equation, the following expression can be attained:

$$
\sqrt{\frac{M}{4 \gamma}} \int_{0}^{A_{0}} \frac{d Z}{\sqrt{\left(A_{0}-Z\right) G(Z, \alpha)}}=\int_{0}^{T / 4} d t
$$

where $Z=A_{0} \sin ^{2} \phi$. Finally, the period of the oscillatory motion can be achieved as follows:

$$
T=4 \sqrt{\frac{M A_{0}}{\gamma}} \int_{0}^{\pi / 2} \frac{\sin \phi d \phi}{\sqrt{G\left(A_{0} \sin ^{2} \phi, \alpha\right)}},
$$

from which the oscillation frequency can be obtained by:

$$
f=\frac{1}{4 \sqrt{\frac{M A_{0}}{\gamma} \int_{0}^{\pi / 2} \frac{\sin \phi d \phi}{\sqrt{G\left(A_{0} \sin ^{2} \phi, \alpha\right)}}}} .
$$

\section{Results and discussion}

In this section, first of all, numerical results are presented for the vdW potential energy and interaction force of telescope nanothermometer with finite tubes. Thereafter, based on the formulation presented in Section 4, results of oscillatory frequency for the shuttle and telescope nanothermometers are proposed. All the constants used in the numerical calculations are given in Table 1.

\section{1. vdW interactions for telescope configuration with finite tube}

Alterations to the vdW potential energy and interaction force with respect to axial distance $Z$ are respectively illustrated in Figure 4(a) and (b) for different half lengths of inner tube. In this figure, the left-hand side tube is a $(10,10) \mathrm{CNT}$, the inner tube is a $(5,5)$ $\mathrm{CNT}$, and the right-hand side one is a $(12,12) \mathrm{CNT}$.

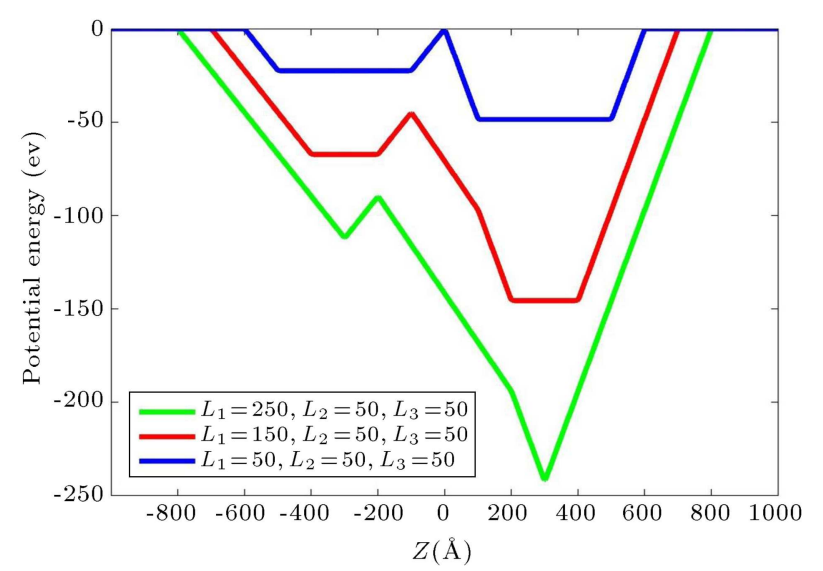

(a)

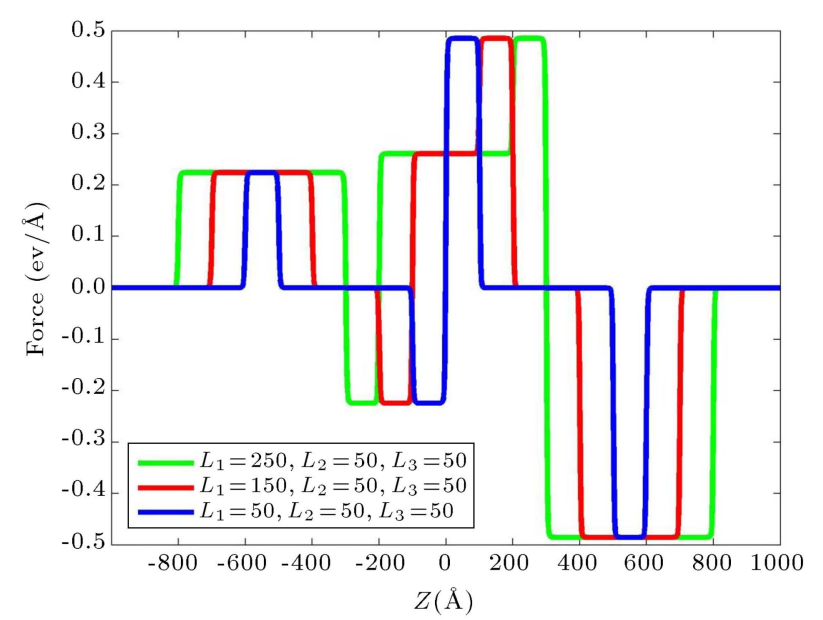

(b)

Figure 4. Variations of the vdW interactions with respect to axial distance $Z$ for telescope configuration with finite tubes: (a) Potential energy and (b) interaction force; $R_{1}=3.392 \AA, R_{2}=6.784 \AA, R_{3}=8.142 \AA, H=500 \AA$, and $h=500 \AA$.

Table 1. Constants used in the numerical calculations.

\begin{tabular}{lc}
\hline${ }^{*} A$ (attractive constant for graphene-graphene) & $15.2\left(\mathrm{eV} \AA^{6}\right)$ \\
${ }^{*} B$ (repulsive constant for graphene-graphene) & $24.1 \times 10^{3}\left(\mathrm{eV}^{12}\right)$ \\
${ }^{*} n_{c}$ (atom density for a graphene) & $0.3812 \AA^{-2}$ \\
Radius of $(5,5) \mathrm{CNT}$ & $3.392 \AA$ \\
Radius of $(10,10) \mathrm{CNT}$ & $6.784 \AA$ \\
Radius of $(12,12) \mathrm{CNT}$ & $8.142 \AA$ \\
\hline
\end{tabular}

*From [10] 
This figure shows that the potential energy between the right-hand side nanotube and the inner CNT is greater than the level of potential energy between the left-hand side and the inner CNTs. This observation is due to the reason that the inter-wall spacing between the inner CNT and the right-hand side CNT is smaller compared to the interwall spacing between the inner CNT and left-hand side CNT. Additionally, the profile of potential energy is seen to be sensitive to the changes in $L_{1}$. For the case where the distance between the two ends of outer CNTs is kept constant, as $L_{1}$ increases, the potential energy decreases and its profile changes from a trapezoid shape to a V-shape. But, from Figure 4(b), it can be observed that the minimum or maximum of the vdW interaction force is not affected by the length of the inner tube.

Exhibited in Figure 5(a) and (b) are the variations of the vdW potential energy and interaction force with

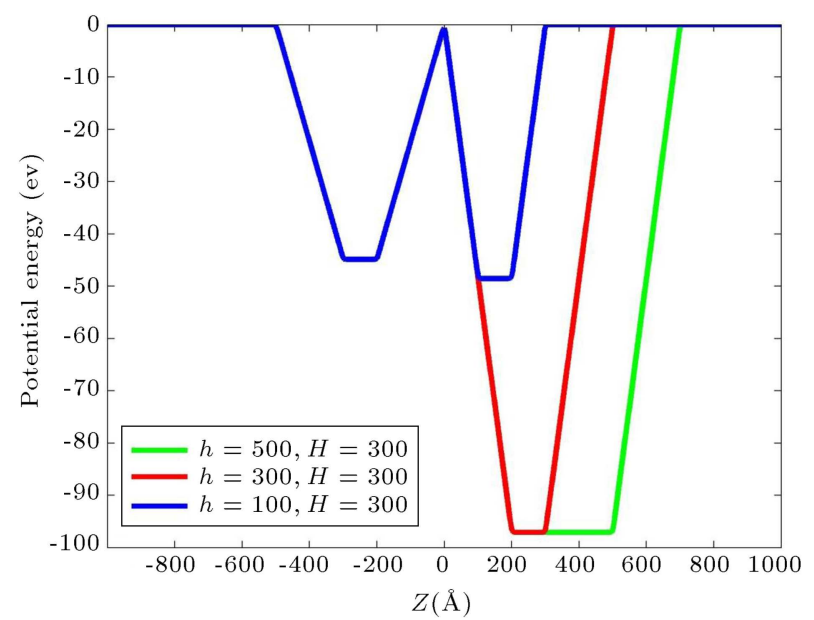

(a)

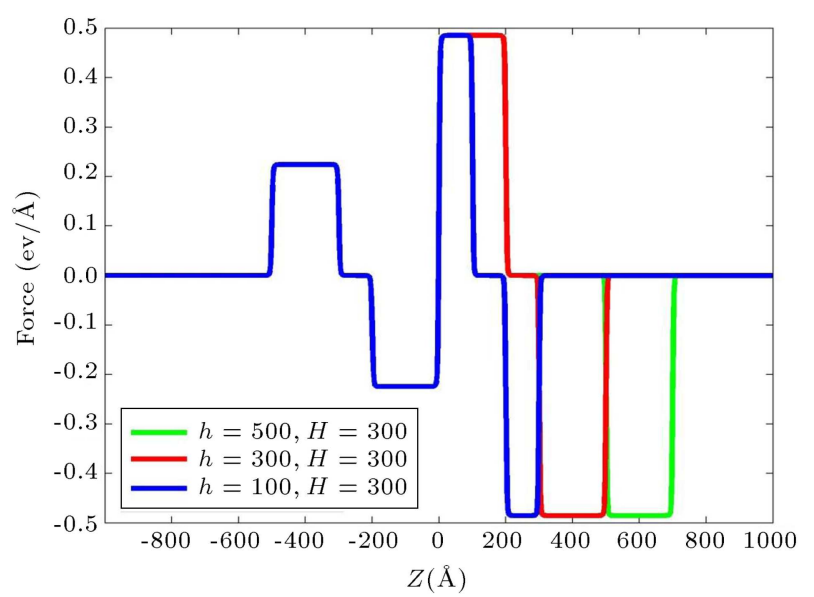

(b)

Figure 5. Variations of the vdW interactions with respect to axial distance $Z$ for telescope configuration with finite tubes: (a) Potential energy and (b) interaction force; $R_{1}=3.392 \AA, R_{2}=6.784 \AA, R_{3}=8.142 \AA$, and $L_{1}=L_{2}=L_{3}=100 \AA$. respect to axial distance $Z$ for various $h$. It is noted that the lengths of right- and left-hand side CNTs are denoted by $h$ and $H$, respectively, as shown in Figure 1. From Figure 5(a), one can deduce that when $h$ alters, only the potential energy attributed to the interaction of inner CNT and the right-hand side CNT changes.

Effect of increasing the length of the left-hand side CNT on the alteration of the vdW potential energy and interaction force is depicted in Figures 6(a) and 6(b). By compering these figures with Figures 5(a) and 5(b), it is seen that the effect of increasing $H$ on the potential energy and interaction force of the left-hand side CNT and the inner CNT is the same as that of increasing $h$ on the vdW interactions of the right-hand side and inner CNT.

Figure 7 shows the prediction of the vdW potential energy and interaction force using only the dominant terms of the $\mathrm{vdW}$ interaction equations. This figure clearly exhibits that the terms consisting of arctangent and $G_{b}^{(k)}$ multipliers in Eqs. (22) and (25) can approximate the vdW potential energy and interaction force with good accuracy.

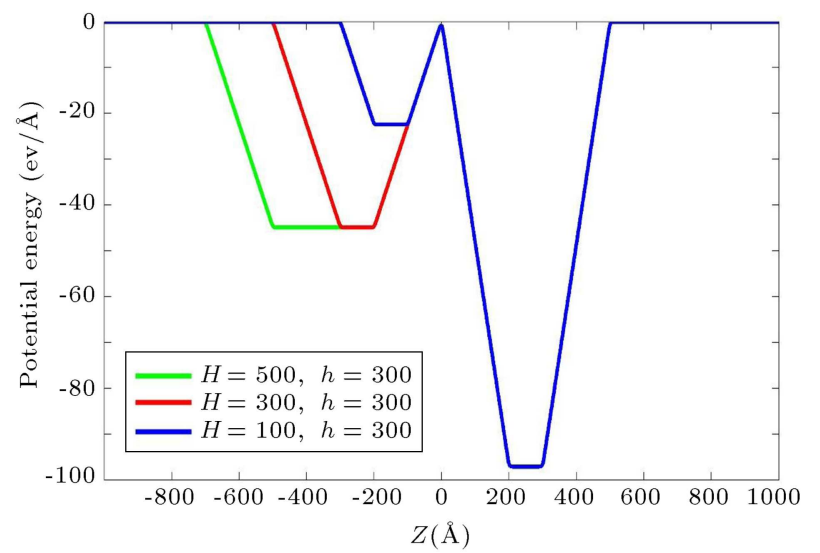

(a)

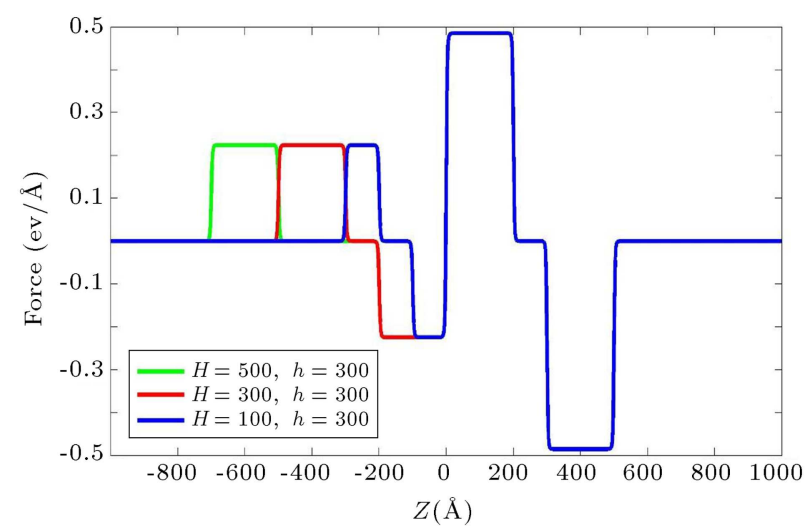

(b)

Figure 6. Variations of the vdW interactions with respect to axial distance $Z$ for telescope configuration with finite tubes: (a) Potential energy and (b) interaction force; $R_{1}=3.392 \AA, R_{2}=6.784 \AA, R_{3}=8.142 \AA$, and $L_{1}=L_{2}=L_{3}=100 \AA$. 


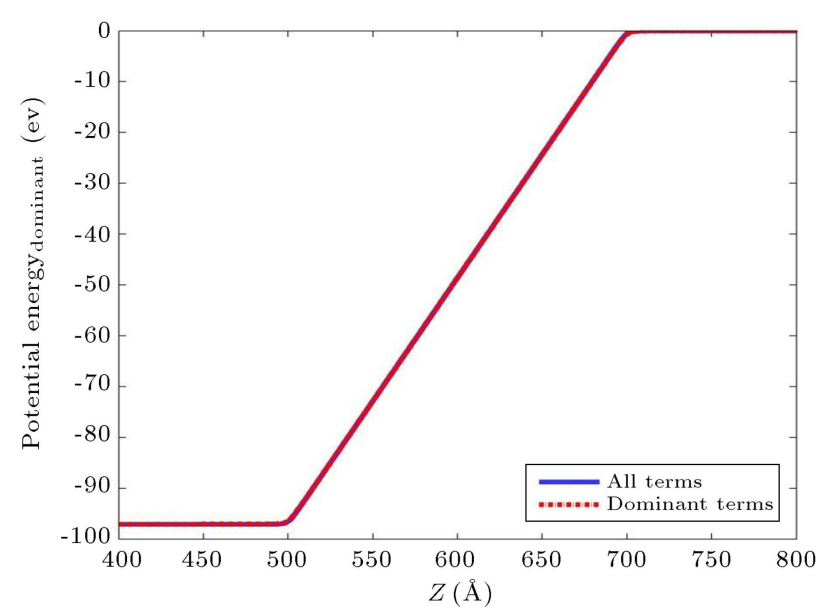

(a)

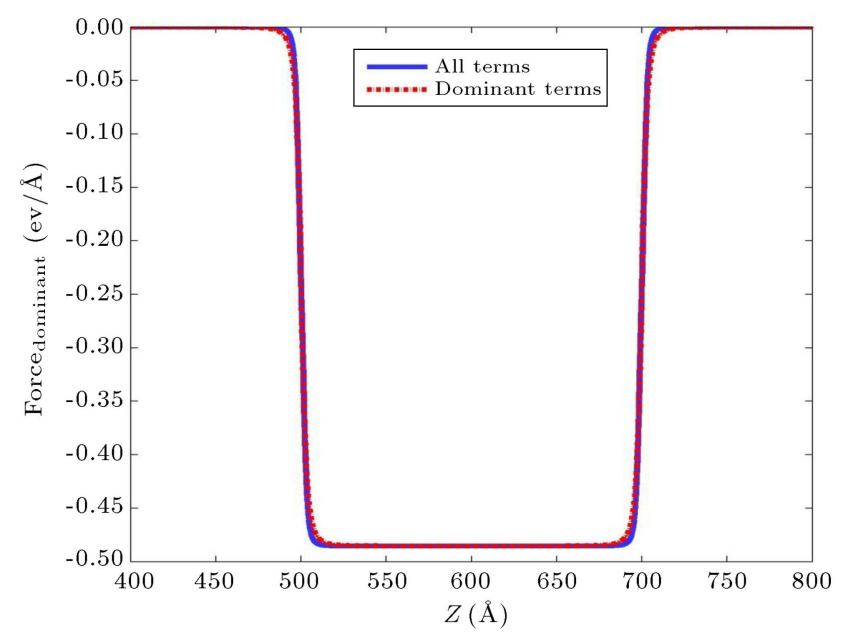

(b)

Figure 7. The effect of dominant terms on the distributions of the vdW interactions: (a) Potential energy and (b) interaction force; $R_{1}=3.392 \AA, R_{2}=6.784 \AA$, $R_{3}=8.142 \AA, L_{1}=L_{2}=L_{3}=100$ Aand $H=h=500 \AA$.

\subsection{Oscillatory frequency}

The frequency profiles of shuttle and telescope configurations are illustrated in Figures 8 and 9, respectively. These figures exhibit that the frequency of oscillation, which is in the $\mathrm{GHz}$ range, decreases as the amplitude increases. The effect of the outer tube length on the frequency behavior is shown in Figure 8. As mentioned earlier, in the shuttle configuration, the outer tube oscillates. According to this figure, it is observed that as the length of the outer CNT increases, the frequency of shuttle configuration decreases. This is expected because by increasing the length of the CNT, the mass increases which slows down the tube movement. In the telescope configuration, as it was stated earlier, the inner tube is movable, while the other two outer CNTs are fixed. Based on Figure 9, it can be deduced that increasing the length of movable tube has similar effect on the frequency of oscillation as it was observed for the shuttle configuration.

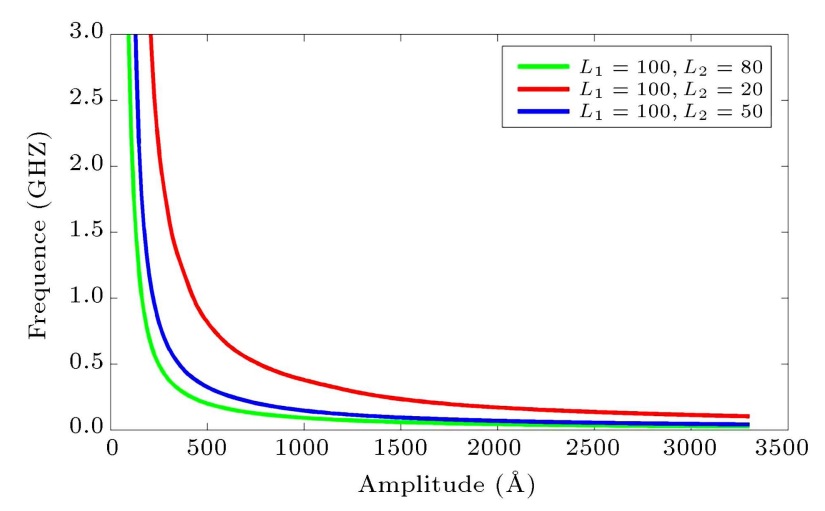

Figure 8. Frequency-amplitude curves for shuttle configurations, $b_{1}=2.035 \AA, b_{2}=6.784 \AA$.

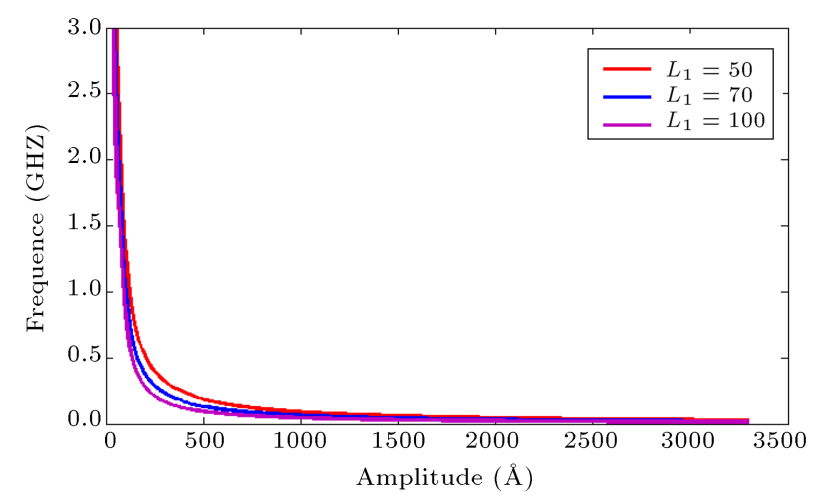

Figure 9. Frequency-amplitude curves for telescope configurations; $L_{1}=50 \AA, L_{3}=20 \AA, H=100 \AA$, $h=100 \AA, b_{1}=4.071, b_{2}=6.784 \AA$, and $b_{3}=10.846 \AA$.

\section{Conclusion}

The aim of the present paper is to obtain the oscillatory frequencies of CNT-based nanothermometers. In order to derive the equation of motion, the vdW interaction force between constituent CNTs of nanothermometer must be used. To this end, upon the utilization of the Lennard-Jones potential function together with the continuum approximation, vdW potential energy and interaction force for telescope configurations were obtained in terms of a single integral. After solving the equation of motion, a semi-analytical expression was obtained which can be readily used to evaluate the frequency of oscillation. This new expression enables us to study the effect of different system parameters on the dynamic behavior of such $\mathrm{GHz}$ oscillators. Results show that the geometrical parameters, such as length of movable tube, affect the oscillatory behavior of such systems.

\section{References}

1. Iijim, S. "Helical microtubules of graphitic carbon", Nature, 354(6348), pp. 56-58 (1991).

2. Bafekrpour, E., Salehi, M., Sonbolestan, E. and 
Fox, B. "Effects of micro-structural parameters on mechanical properties of carbon nanotube polymer nanocomposites", Scientia Iranica, 21(2), pp. 403-413 (2014).

3. Fallah, M., Faez, R. and Jafari, A.H. "Simulation of carbon nanotube field effect transistor with two different gate insulator", Scientia Iranica, 20(6), pp. 2332-2340 (2013).

4. Ansari, R., Sadeghi, S. and Ajori, S. "Continuum and molecular dynamics study of C60 fullerene-carbon nanotube oscillators", Mech. Res. Commun., 47, pp. 18-23 (2013).

5. Ansari, R., Sadeghi, F. and Motevalli, B. "A comprehensive study on the oscillation frequency of spherical fullerenes in carbon nanotubes under different system parameters", Commun. Nonlinear Sci. Numer. Simul., 18(3), pp. 769-784 (2013).

6. Ansari, R., Sadeghi, F. and Alipour, A. "Oscillation of C60 fullerene in carbon nanotube bundles", J. Vib. Acoust., 135(5), p. 051009 (2013).

7. Dong, J. "Ultrafast all-optical signal processing based on single semiconductor optical amplifier and optical filtering", IEEE J. Sel. Top. Quantum Electron, 14(3), pp. $770-778$ (2008).

8. Fan, Z., Tao, X., Cui, X., Fan, X., Zhang, X. and Dong, L. "Metal-filled carbon nanotube based optical nanoantennas: bubbling, reshaping, and in situ characterization", Nanoscale, 4(18), pp. 5673-5679 (2012).

9. Cumings, J. and Zettl, A. "Low- friction nanoscale linear bearing realized from multiwall carbon nanotubes", Science, 289(5479), pp. 602-604 (2000).

10. Girifalco, L.A., Hodak, M. and Lee, R.S. "Carbon nanotubes, buckyballs, ropes, and a universal graphitic potential", Phys. Rev. B, 62(19), pp. 13104-13110 (2000).

11. Ansari, R. and Motevalli, B. "On new aspects of nested carbon nanotubes as Gigahertz oscillators", J. Vib. Acoust, 133(5), pp. 1-10 (2011).

12. Ansari, R., Alipour, A. and Sadeghi, F. "Oscillatory characteristics of carbon nanotubes inside carbon nanotube bundles", J. Appl. Phys., 112(2012), p. 124310 (2012).

13. Ansari, R., Mahmoudinezhad, E., Alipour, A. and Hosseinzadeh, M. "A comprehensive study on the encapsulation of methane in single-walled carbon nanotubes", J. Comput. Theor. Nanosci, 10(9), pp. 22092215 (2013).

14. Ansari, R. and Hosseinzadeh, M. "Mechanics of singlewalled carbon nanotubes inside open single-walled carbon nanocones", J. Mech. Sci. Technol., 27(11), pp. 3363-3370 (2013).

15. Ansari, R. and Sadeghi, F. "On the mechanics of C60 fullerene inside open carbon nanocones: A continuum study", Physica E, 69, pp. 1-12 (2015).

16. Ansari, R. and Sadeghi, F. "Mechanics of nested spherical fullerenes inside multi-walled carbon nanotubes", Eur. J. Mech. A-Solid, 49, pp. 283-292 (2015).
17. Ansari, R., Sadeghi, F. and Faghih Shojaei, M. "On the mechanics of ellipsoidal fullerenes inside open carbon nanocones: A novel numerical approach", Nano, 9(3), p. 1450034 (2014).

18. Ganzhorn, M., Klyatskaya, S., Ruben, M. and Wernsdorfer, W. "Strong spin-phonon coupling between a single-molecule magnet and a carbon nanotube nanoelectromechanical system", Nature Nanotechnology, 8(3), pp. 165-169 (2013).

19. Holek, S., Reguera, D. and Rubi, J.M. "Carbonnanotube-based motor driven by a thermal gradient", J. Phys. Chem. C, 117(6), pp. 3109-3013 (2013).

20. Hall, A.R., Paulson, S., Cui, T., Lu, J.P., Qin, L.C. and Washburn, S. "Torsional electromechanical systems based on carbon nanotubes", Rep. Prog. Phys., 75(11), p. 116501 (2012).

21. Ganzhorn, M. and Wernsdorfer, W. "Dynamics and dissipation induced by single-electron tunneling in carbon nanotube nanoelectromechanical systems", Phys. Rev. Lett., 108(17), p. 175502 (2012).

22. Poetschke, M., Rocha, C.G., Torres, L.E.F.F., Roche, S. and Cuniberti, G. "Modeling graphene-based nanoelectromechanical devices", Phys. Rev. B, 81(19), p. 193404 (2010).

23. Wu, C.C. and Zhong, Z. "Capacitive spring softening in single-walled carbon nanotube nanoelectromechanical resonators", Nano Lett., 11(4), pp. 1448-1451 (2011).

24. Li, Y.B., Bando, Y., Golberg, D. and Liu, Z.W. "Ga-filled single-crystalline $\mathrm{MgO}$ nanotube: Widetemperature range nanothermometer", Appl. Phys. Lett., 83(5), pp. 999-1001 (2009).

25. Vetrone, F., Naccache, R., Zamarrón, A., Fuente, A.J., Sanz-Rodríguez, F., Maestro, L.M., Rodriguez, E.M., Jaque, D., Solé, J.G. and Capobianco, J.A. "Temperature Sensing Using Fluorescent Nanothermometers", ACS Nano, 4(6), pp. 3254-3258 (2010).

26. Tashiro, R. and Sugiyama, H. "A nanothermometer based on the different $\pi$ stackings of B- and Z-DNA", Angew. Chem. Int. Ed. Engl., 42(48), pp. 6018-6020 (2003).

27. Liu, Z., Bando, Y., Hu, J., Ratinac, K. and Ringer, S.P. "A novel method for practical temperature measurement with carbon nanotube nanothermometers", Nanotechnology, 17(15), p. 3681 (2006).

28. Lan, Y., Wang, H., Chen, X., Wang, D., Chen, G. and Ren, Z. "Nanothermometer using single crystal silver nanospheres", Adv. Mater., 21(47), pp. 49394844 (2009).

29. Popov, A.M., Bichoutskaia, E., Lozovik, Y.E. and Kulish, A.S. "Nanoelectromechanical systems based on multi-walled nanotubes: nanothermometer, nanorelay, and nanoactuator", Phys. Status Solidi (a), 204(6), pp. 1911-1917 (2007).

30. Gao, Y., Bando, Y., Liu, Z., Golberg, D. and Nakanishi, H. "Temperature measurement using a galliumfilled carbon nanotube nanothermometer", Appl. Phys. Lett., 83(14), p. 2913 (2003). 
31. Liu, Z., Bando, Y., Mitome, M. and Zhan, J. "Unusual freezing and melting of gallium encapsulated in carbon nanotubes", Phys. Rev. Lett., 93(9), pp. 095504095508 (2004).

32. Liu, X., Tang, X., Hou, Y., Wua, Q. and Zhang, G. "Fluorescent nanothermometers based on mixed shell carbon nanodots", RSC Adv., 5(99), pp. 81713-81722 (2015).

33. Pi, S., Zhong, J., Wei, K. and Liu, W. "Optimal anti-noise ability and high sensitivity in magnetic nanothermometry", IEEE Trans Nanotechnol., 15(3), pp. 409-415 (2016).

34. Mayle, S., Tanuj Gupta, T., Davis, S., Chandrasekhar, V. and Shafraniuk, S. "Thermometry and thermal management of carbon nanotube circuits", J. Appl. Phys., 117(19), p. 194305 (2015)

35. Su, J., Sun, M., Zhang, X., Huang, Y. and Gao, Y. "Ga filled nanothermometers with high sensitivity and wide measuring range", J. Nanosci. Nanotechnol., 12(8), pp. 6397-6400 (2012).

36. Zhou, H., Sharma, M., Berezin, O., Zuckerman, D. and Berezin, M.Y. "Nanothermometry: From microscopy to thermal treatments", ChemPhysChem, 17(1), pp. 27-36 (2015).

37. Bichoutskaia, E., Popov, A.M., Lozovik, Y.E., Ivanchenko, G.S. and Lebedev, N.G. "Electromechanical nanothermometer", Phys. Lett. A, 366(4), pp. 480486 (2007).

38. Rahmat, F., Thamwattana, N. and Hill, J.M. "Carbon nanotube oscillators for applications as nanothermometers", J. Phys. A: Math. Theor, 43(40), pp. 405209-405234 (2010).
39. Ansari, R., Daliri, M. and Hosseinzadeh, M. "On the van der Waals interaction of carbon nanotubes as electromechanical nanothermometers", Acta Mech. Sinica, 29(4), pp. 622-632 (2013).

\section{Biographies}

Reza Ansari received his PhD from the University of Guilan, Iran in 2008. During his PhD studies, he was also a visiting fellow at Wollongong University in Australia from 2006 to 2007. He is currently a faculty member of the Department of Mechanical Engineering, University of Guilan. He authored more than 100 journal articles and 12 book chapters. His research background and interests include computational nano/micro-mechanics, advanced numerical techniques, nonlinear analyses, and prediction of the mechanical behavior of smart composite/FGM shelltype structures.

Maryam Daliri received her MSc from the Pardis University of Guilan, Iran, in 2016. She authored one journal article. Her research background and interest is computational nanomechanics.

Mohadeseh Hosseinzadeh received her MSc from the University of Guilan, Iran in 2013. She is currently a faculty member of the Department of Mechanical Engineering, Mooj institute of higher education. She authored 7 journal articles. Her research background and interests include computational nanomechanics and prediction of the mechanical behavior of FGM shell-type structures. 